\title{
Microvascular injury and hypoxic damage: emerging neuropathological signatures in COVID-19
}

\author{
Zane Jaunmuktane ${ }^{1,2} \cdot$ Ula Mahadeva $^{3} \cdot$ Anna Green $^{3} \cdot$ Vivek Sekhawat $^{3} \cdot$ Nicholas A. Barrett $^{4,5} \cdot$ Lucy Childs $^{6}$. \\ Manu Shankar-Hari ${ }^{4,7} \cdot$ Maria Thom ${ }^{1,8} \cdot$ Hans Rolf Jäger ${ }^{9,10} \cdot$ Sebastian Brandner ${ }^{1,11}$ (B)
}

Received: 15 June 2020 / Revised: 1 July 2020 / Accepted: 2 July 2020 / Published online: 8 July 2020

(c) The Author(s) 2020

In patients with COVID-19, neurological complications are increasingly recognised, but only few neuropathological studies are available, documenting microthrombi and acute infarcts [1], hypoxic changes with no specific pathology [9] or perivascular lymphocytic infiltration in brainstem [10]. We report here neuropathology of two COVID-19 patients with findings in one strikingly similar to those described in a recent case report, with neocortical infarcts and small haemorrhagic and non-haemorrhagic white matter lesions [8], suggesting an emerging pattern of characteristic alterations, also observed radiologically [3].

Clinical data for both patients are provided in supplementary Fig. 1 and neuropathology and imaging in Figs. 1, 2 and supplementary Figs. 2, 3, 4.

Patient 1, a male in his fifties, suffered from cardiac arrest, shortly before veno-venous extracorporeal membrane

Electronic supplementary material The online version of this article (https://doi.org/10.1007/s00401-020-02190-2) contains supplementary material, which is available to authorized users.

Zane Jaunmuktane

z.jaunmuktane@ucl.ac.uk

$\triangle$ Sebastian Brandner

s.brandner@ucl.ac.uk

1 Division of Neuropathology, National Hospital for Neurology and Neurosurgery, University College London NHS Foundation Trust, London, UK

2 Department of Clinical and Movement Neurosciences and Queen Square Brain Bank for Neurological Disorders, Queen Square Institute of Neurology, University College London, London, UK

3 Department of Cellular Pathology, Guy's and St Thomas' NHS Foundation Trust, London, UK

4 Department of Intensive Care Medicine, Guy's and St Thomas' NHS Foundation Trust, London, UK

5 Faculty of Life Sciences and Medicine, King's College London, London, UK oxygenation (vvECMO) and succumbed to multifocal brain infarcts. The ischaemic lesions in watershed areas are in keeping with sustained hypotension during cardiac arrest. The exact cause of the large right middle cerebral artery (MCA) and the bilateral posterior (PCA) infarcts remains uncertain. Thromboembolic origin from the known pulmonary embolus is excluded due to closed foramen ovale. The infarcts may be due to local thrombosis or, similar to watershed infarcts, may have developed as a complication of protracted hypotension during cardiac arrest. The florid leukocytoclastic reaction in the infarcts may simply be a reaction to reperfusion injury but may also be due to augmented immune response.

The second patient, a female in her sixties, was intubated and ventilated, but after discontinuing sedation, remained unresponsive and died due to multiorgan failure. The bilateral pallidal infarcts most likely were caused by hypoxia. Possible pathogeneses for the cortical and white matter microlesions, including microbleeds, include viral infection-related vascular injury; immune-mediated; or hypoxia

6 Department of Radiology, Guy's and St Thomas' NHS Foundation Trust, London, UK

7 School of Immunology and Microbial Sciences, King's College London, London, UK

8 Department of Clinical and Experimental Epilepsy, Queen Square Institute of Neurology, University College London, London, UK

9 Lysholm Department of Neuroradiology, National Hospital for Neurology and Neurosurgery, University College London NHS Foundation Trust, London, UK

10 Neuroradiological Academic Unit, Department of Brain Repair and Rehabilitation, Queen Square Institute of Neurology, University College London, London, UK

11 Department of Neurodegenerative Disease, Queen Square Institute of Neurology, University College London, London, UK 

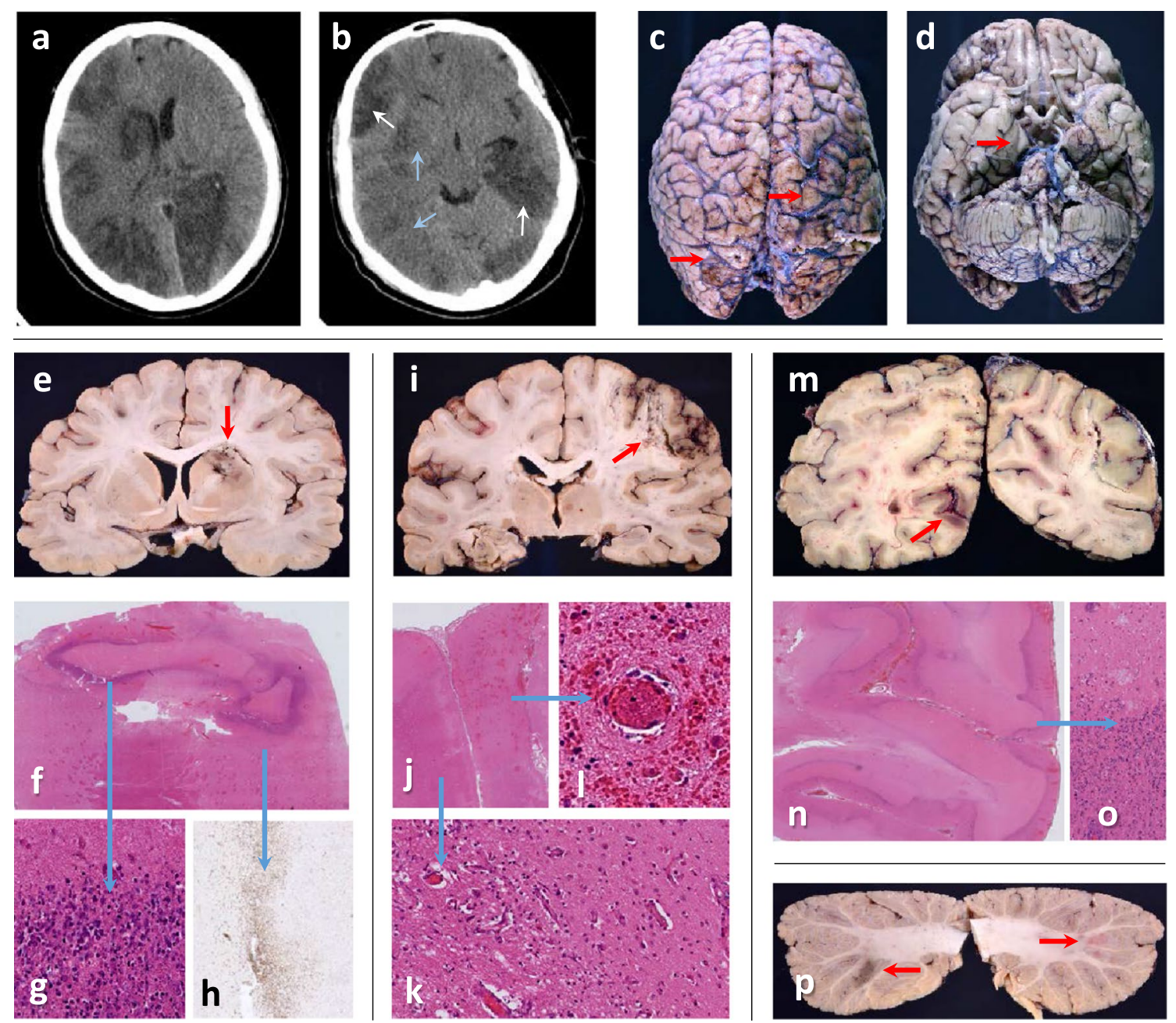

Fig. 1 Case 1: a, b Head-CT shows recent (blue arrows) and established (white arrows) multifocal infarcts. $\mathbf{c}$ Rusty discolouration (red arrows) on brain surface. d Right uncal grooving (red arrow). e Bilateral acute and subacute watershed infarcts in the anterior-MCA and MCA-PCA territories and a subacute infarct in the right lentiform nucleus (red arrow). f, $\mathbf{g}$ Dense inner rim of degenerating neutrophils (blue arrow) and $\mathbf{h}$, an outer rim (blue arrow) of macrophages (CD68). i, j Macroscopy and microscopy of confluent infarcts across

secondary to hypotension, local thrombosis, or thromboemboli. MRI-pathology correlation showed that leptomeningeal hyperintensity corresponded to lymphohistiocytic inflammation. Microglial nodules, neuronophagia and vascular injury, including signs of vasculitis, distant from the infarcts were not identified in either case and inflammation in the medulla was similar to patients with a variety of other neurological diseases (supplementary Table 1, supplementary Fig. 4).

Several mechanisms for the SARS-CoV-2-related neurological complications are plausible. First, direct viral invasion via haematogenous or retrograde axonal route with intracellular accumulation either in endothelial cells, smooth muscle cells, pericytes, inflammatory cells (particularly macrophages), neurones or glial cells. Second, an the right MCA territory (acute and subacute, red arrow). k Infarcts are ischaemic, with granulation tissue and macrophages (blue arrow), or $\mathbf{l}$, with perivascular haemorrhages and fibrin thrombi (blue arrow). m Bilateral acute and subacute infarcts in the PCA territories (both occipital lobes (red arrow), and $\mathbf{i}$ left hippocampus and thalamus. $\mathbf{n}$, o Frequently, subacute infarcts show prominent leukocytoclastic reaction (blue arrow). p Multiple subacute cortical infarcts in both cerebellar hemispheres (red arrows)

indirect process resulting from hypercoagulability-related thromboembolism or thrombus formation within the brain or an exaggerated cytokine/immune-mediated response to viral infection causing damage to blood vessel walls or cells in the brain.

Viral components specifically in endothelial cells have been documented in kidney [7], lung and skin [5], but not with certainty in brain, although viral RNA of uncertain replicative and infective potential has been detected in the CSF [6] and brain tissue homogenates [7].

The few cases reported to date highlight the complexity of neuropathology in COVID-19 and the difficulty in untangling primary from secondary hypoxic/anoxic changes and iatrogenic aetiologies and suggest that a combination of 

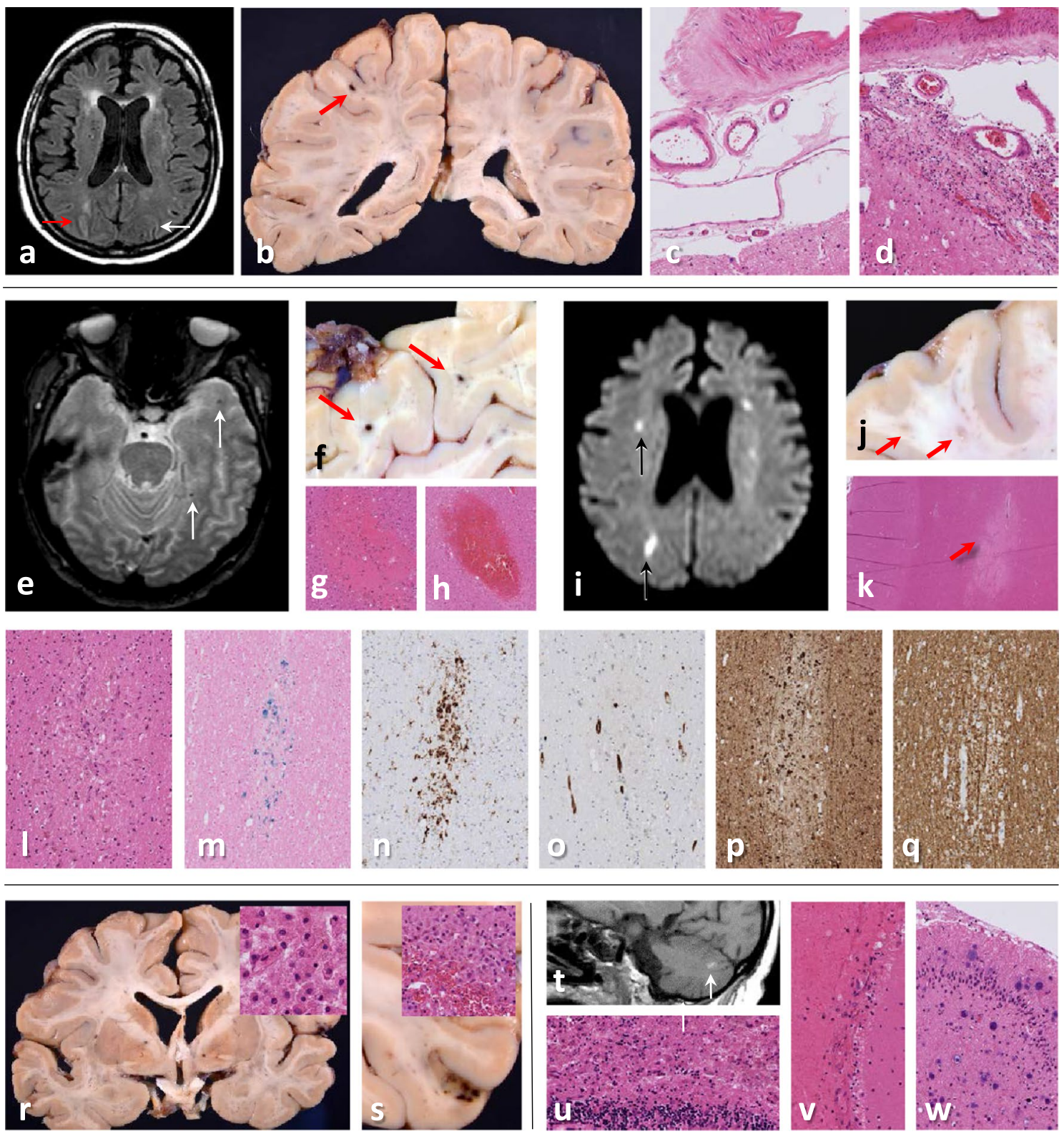

Fig. 2 Case 2: a MRI brain (FLAIR image) shows leukoaraiosis and high signal intensity in the right (red arrow) but not left (white arrow) intraparietal sulcus. b Microhaemorrhage on the left (red arrow), but no gross leptomeningeal pathology. c, d Histology shows mild leptomeningeal lymphohistiocytic inflammation in the right (d), but not left (c) intraparietal sulcus. e Arrows point to microhaemorrhages (T2*weighted MRI), f macroscopically (red arrows) and $\mathbf{g}, \mathbf{h}$ microscopically corresponding to acute and subacute white matter microbleeds. i Small acute infarcts (DWI MRI, black arrows), j macroscopically corresponding to white matter lesions ( $\varnothing 1-4 \mathrm{~mm}$, red arrows). $\mathbf{k}$ Histologically, some are classic acute and subacute microinfarcts (red arrow), $\mathbf{l}$ whilst others contain haemosiderin-laden macrophages, $\mathbf{m}$ shown with Perls staining. n CD68 accentuates many more lesions

different events, rather than a single mechanism, accounts for the various neurological complications, even within one patient. than evident macroscopically or on MRI. o Unremarkable blood vessels are seen within some of the white matter microlesions (CD34 immunostaining). p Swollen axons (indicating damage) on neurofilament (SMI31) immunostaining $\mathbf{q}$ but no demyelination (myelin basic protein (SMI94) immunostaining). r Macroscopic and microscopic examination (inset in $\mathbf{r}$ shows macrophage-rich necrosis) reveal bilateral subacute pallidal infarcts. $\mathbf{s}$ Occasional subacute microinfarcts in the cortex $(\varnothing 1-5 \mathrm{~mm})$, some with haemorrhagic transformation (inset in s). $\mathbf{t}$ High MRI T1 signal foci (white arrow) in the cerebellum correspond histologically to $\mathbf{u}$ subacute infarct, $\mathbf{v}$ fresh leptomeningeal haemorrhages and (not shown) non-haemorrhagic white matter microlesions. w Several chronic infarcts and microinfarcts (likely embolic) in cerebellar cortex and right thalamus (not shown)

It is striking that ACE2 expression is increased in ischaemic brains and also in blood vessels in patients with diabetes [2], given that ACE2 represents the receptor by which 
SARS-CoV-2 enters host cells. Similarly, certain treatment regimens, such as ECMO, may increase the risk of neurological complications [4]. We provide further neuropathological correlates to a radiological feature of subcortical white matter microvascular lesions, including microhaemorrhages. Through future neuropathological studies, it is hoped that the mechanisms leading to tissue damage in COVID-19 will continue to be elucidated, to enable timely and appropriate treatment options.

Acknowledgements The authors would like to thank the patients and their families and the contribution of numerous local physicians to the patients' care and investigation. We also are grateful to Ms Tina Mackay (Senior Anatomical Pathology Technologist) and the other mortuary staff at Guy's and St Thomas' NHS Foundation Trust for their skills contributing to post-mortem examination and care of the deceased, and the biomedical staff of the Division of Neuropathology for their excellent histological assistance. We acknowledge the critical care multidisciplinary team at Guy's and St Thomas' NHS Foundation Trust.

Funding ZJ, MT and SB are supported by the Department of Health's NIHR Biomedical Research Centre's funding scheme to UCLH. Dr. Shankar-Hari is supported by the National Institute for Health Research Clinician Scientist Award (CS-2016-16-011). The views expressed in this publication are those of the author(s) and not necessarily those of the NHS, the National Institute for Health Research or the Department of Health and Social Care.

\section{Compliance with ethical standards}

Conflict of interest The authors declare no conflict of interest.

Open Access This article is licensed under a Creative Commons Attribution 4.0 International License, which permits use, sharing, adaptation, distribution and reproduction in any medium or format, as long as you give appropriate credit to the original author(s) and the source, provide a link to the Creative Commons licence, and indicate if changes were made. The images or other third party material in this article are included in the article's Creative Commons licence, unless indicated otherwise in a credit line to the material. If material is not included in the article's Creative Commons licence and your intended use is not permitted by statutory regulation or exceeds the permitted use, you will need to obtain permission directly from the copyright holder. To view a copy of this licence, visit http://creativecommons.org/licenses/by/4.0/.

\section{References}

1. Bryce C, Grimes Z, Pujadas E, Ahuja S, Beasley MB, Albrecht R, Hernandez T, Stock A, Zhao Z, Al Rasheed M et al (2020)
Pathophysiology of SARS-CoV-2: targeting of endothelial cells renders a complex disease with thrombotic microangiopathy and aberrant immune response. The Mount Sinai COVID-19 autopsy experience. medRxiv: 2020.2005.2018.20099960. https://doi. org/10.1101/2020.05.18.20099960

2. Choi JY, Lee HK, Park JH, Cho SJ, Kwon M, Jo C, Koh YH (2020) Altered COVID-19 receptor ACE2 expression in a higher risk group for cerebrovascular disease and ischemic stroke. Biochem Biophys Res Commun. https://doi.org/10.1016/j. bbrc.2020.05.203

3. Kremer S, Lersy F, de Sèze J, Ferré JC, Maamar A, Carsin-Nicol B, Collange O, Bonneville F, Adam G, Martin-Blondel G et al (2020) Brain MRI Findings in Severe COVID-19: A Retrospective Observational Study. Radiology: 202222. https://doi.org/10.1148/ radiol.2020202222

4. Lorusso R, Gelsomino S, Parise O, Di Mauro M, Barili F, Geskes G, Vizzardi E, Rycus PT, Muellenbach R, Mueller T et al (2017) Neurologic injury in adults supported with veno-venous extracorporeal membrane oxygenation for respiratory failure: findings from the extracorporeal life support organization database. Crit Care Med 45:1389-1397. https://doi.org/10.1097/ccm.00000 00000002502

5. Magro C, Mulvey JJ, Berlin D, Nuovo G, Salvatore S, Harp J, Baxter-Stoltzfus A, Laurence J (2020) Complement associated microvascular injury and thrombosis in the pathogenesis of severe COVID-19 infection: A report of five cases. Translational research : the journal of laboratory and clinical medicine 220:1-13. https ://doi.org/10.1016/j.trsl.2020.04.007

6. Moriguchi T, Harii N, Goto J, Harada D, Sugawara H, Takamino J, Ueno M, Sakata H, Kondo K, Myose N et al (2020) A first case of meningitis/encephalitis associated with SARS-Coronavirus-2. Int J Infect Dis IJID Off Publ Int Soc Infect Dis 94:55-58. https:// doi.org/10.1016/j.ijid.2020.03.062

7. Puelles VG, Lütgehetmann M, Lindenmeyer MT, Sperhake JP, Wong MN, Allweiss L, Chilla S, Heinemann A, Wanner N, Liu $\mathrm{S}$ et al (2020) Multiorgan and renal tropism of SARS-CoV-2. N Engl J Med. https://doi.org/10.1056/NEJMc2011400

8. Reichard RR, Kashani KB, Boire NA, Constantopoulos E, Guo Y, Lucchinetti CF (2020) Neuropathology of COVID-19: a spectrum of vascular and acute disseminated encephalomyelitis (ADEM)like pathology. Acta Neuropathol. https://doi.org/10.1007/s0040 1-020-02166-2

9. Solomon IH, Normandin E, Bhattacharyya S, Mukerji SS, Keller K, Ali AS, Adams G, Hornick JL, Padera RF, Sabeti P (2020) Neuropathological Features of Covid-19. N Engl Journal Med. https://doi.org/10.1056/NEJMc2019373

10. von Weyhern CH, Kaufmann I, Neff F, Kremer M (2020) Early evidence of pronounced brain involvement in fatal COVID-19 outcomes. Lancet (Lond Engl). https://doi.org/10.1016/s0140 $-6736(20) 31282-4$

Publisher's Note Springer Nature remains neutral with regard to jurisdictional claims in published maps and institutional affiliations. 